\title{
Fatal Disseminated Mucormycosis In A Patient With Mantle Cell non-Hodgkin's Lymphoma: An Autopsy Case
}

\author{
Inci Alacacioglu ${ }^{1}$, Aydanur Kargi ${ }^{2}$, Mehmet Ali Ozcan ${ }^{1}$, Ozden Piskin ${ }^{1}$, Cilem Solak ${ }^{1}$, Mustafa Secil ${ }^{3}$, Mehtat Unlu², Fatih \\ Demirkan ${ }^{1}$, Guner Hayri Ozsan ${ }^{1}$, Bulent Undar ${ }^{1}$ \\ ${ }^{1}$ Dokuz Eylul University Faculty of Medicine, Deparment of Hematology; ${ }^{2}$ Dokuz Eylul University Faculty of Medicine, Deparment of \\ Pathology; ${ }^{3}$ Dokuz Eylul University Faculty of Medicine, Department of Radiology; Izmir, Turkey
}

\begin{abstract}
A patient with mantle cell non-Hodgkin's lymphoma presented herself with fever, nausea, right upper quadrant pain on the 7th day of R-CHOP chemotherapy. After hospitalization with the suspicion of acute cholecystitis, she received antibiotherapy with G-CSF because of emerging neutropenia at the 10th day of chemotherapy. Abdominal computed tomography revealed small infarcts in the spleen and kidneys. The echymotic lesion which developed on her right lateral malleolus, became bullous in the following days and treated as ecthyma gangrenosum. Altough the patient was afebrile with a normal neutrophil count on the third day of antibiotherapy, she developed acute renal failure and deteriorated rapidly. The patient underwent hemodialysis but expired on the 10th day of hospitalization. Post mortem autopsy findings showed ischemic infarction and necrosis of parenchyma due to mycotic thrombosis of arteries and veins of many organs (heart, lung, diaphgram, kidneys, spleen, gut mucosa) as well as invasion of vessel walls and parenchyma by mucor. We reviewed mucormycosis in the light of this case.
\end{abstract}

Key-Words: Mucormycosis, lymphoma, autopsy.

Mucormycosis is an invasive fungal infection caused by various members of the class Phycomycetes, especially Mucoracea, subdivided into the genera Absidia, Rhizopus and Mucor. Mucorales represent the third leading cause of invasive fungal infections following Aspergillus and Candida species in many parts of the world [1-4]. Among patients with hematologic disorders, mucormycosis most commonly occurs in those with acute leukemia or lymphoma who have developed neutropenia due to malignancy or to chemotherapy, and in transplanted patients receiving immunosupressive treatment [2,5-7]. Rhino-cerebral, maxillo-facial and pulmonary infections are the most frequent clinical forms, but it may involve multiple organs such as brain, kidneys, liver or gastro-intestinal tract [8]. Neutropenic patients are at a high risk of developing a disseminated mucormycosis [9]. Like Aspergillus, Phycomycetes has a tendency for vascular invasion and tissue necrosis, with resultant hemorrhage and necrotic lesions [8]. The prognosis remains extremely poor [2]. The mainstay of treatment for mucormycosis is early surgical intervention to remove all dead and infected tissue, when feasable, along with intravenous antifungal therapy and neutrophil recovery.

Herein, we report an unusual autopsy case of systemic mucormyosis involving several organs, including heart which developed in a patient with mantle cell lymphoma.

\section{Case report}

A 63-year-old female patient admitted the hospital with fever, nausea, vomiting, loss of appetite and abdominal pain Received on 9 January 2009; revised 6 April 2009.

Address for correspondence: Dr. Inci Alacacioglu, MD. Dokuz Eylul University, Faculty of Medicine. Zip code: 35340 Inciralti / Izmir, Turkey. Phone: +90-232-412 37 30. Fax: +90-232-412 37 19. E-mail: inci074@yahoo.com.

The Brazilian Journal of Infectious Diseases 2009;13(3):238-241. (C) 2009 by The Brazilian Journal of Infectious Diseases and Contexto Publishing. All rights reserved. which started two days ago. She has received first R-CHOP (rituximab-cyclophosphamide, vincristine, doxorubicine, prednisolone) chemotherapy due to leukemic form of mantle cell non-Hodgkin lymphoma seven days before and discharged without complication. On her physical examination, there were tenderness at right upper quadrant of abdomen and petechial lesions on her back. Other system examination was normal. Because of her cholelithiasis history, she was internalized with suspicion of acute cholecystitis.

Her laboratory examinations showed that; white blood cells 9,1 $1 \times 10 \%$ L (53\% polymorphonuclear leukocytes, 45\% lymphocytes, $2 \%$ monocytes), hemoglobin level 11,2 g/dL, platelets $28 \times 10^{9} / \mathrm{L}$, alkaline phosphatase $301 \mathrm{u} / \mathrm{L}$, lactate dehydrogenase (LDH) 505 IU/L, gama-glutamyl transpeptidase (GGT) 45u/L, aspartate aminotransferase (AST) 76u/L, alanine aminotransferase (ALT) $35 \mathrm{u} / \mathrm{L}$. There was no any sign of acute cholecystitis on her abdominal ultrasonography. On her follow-up period, she had a body temperature as $38.1^{\circ} \mathrm{C}$ which falled to normal level itself in one hour. Since at the third day of hospitalization, she became neutropaenic (tenth day of chemotherapy also) and she had a recurrent fever, cefepime (3x2 gr IV), ciprofloxacin (2x200 mg IV) antibiotherapy were immediately initiated with G-CSF support. At the second day of antibiotherapy, an ecymotic lesion developed on her right malleolus. She was still febrile. At the third day of antibiotherapy, ecymotic lesion became bigger and her right ankle became edematous and bullous lesion developed on the right malleolus (Figure 1). Other ecymotic lesions developed on her left plantar surface and on her presacral area. She was consultated with dermatology, rheumotology and orthopedy departments for her lesion on the ankle. There was no bony destruction other than soft tissue swelling at the direct graphy of right ankle. Her platelet level was raised so joint aspiration could be made. Joint aspiration fluid examination revealed leukocyte count $4 \times 10^{9} / \mathrm{L}$ with $71 \%$ neutrophils. On the microscopic examination, there 
were not any microorganisms, cultures were also negatives (blood cultures, joint fluid cultures). At that time, cefepime antibiotherapy was changed with imipenem due to suspicion of ecthyma gangrenosum, pseudomonas infection. She had recurrent complaint of abdominal pain. Her abdominal computed tomography revealed multiple small infarctions at spleen and kidneys, cholelithiasis, multiple intraabdominal lenfadenomegalies (Figure 2). In order to rule out probable infective endocarditis, echochardiography was made but signs of this infection were not seen. At the fourth day of antibiotherapy, she became afebrile and her neutrophile count raised with G-CSF support. She felt good except for pain at her ankle. Two days later, she was begining to deteriorate again. She was consultated with nephrology department for her high creatinine level. Firstly, she was evaluated with the suspicion of acute contrast nephropathy. But due to increasing creatinine level, renal doppler ultrasonography was made. There were not any blood flow at renal arteries and veins. She was taken on hemodialysis programme. But she deteriorated rapidly and died at tenth days of hospitalization. Due to this rapid deterioration although she became afebrile and relieved from neutropaenia, autopsy was made.

Post mortem examination showed ischemic infarction of several organs (heart, lung, diaphgram, kidneys, spleen, gut mucosa, subcutaneus tissues) due to invasion of the walls and occlusion of lumens of arteries and veins by fungal hyphae, morphologically compatible with mucormycosis [Figure 3, Figure 4 (GMS stain)].

\section{Discussion}

Mucormycosis is rare filamentous fungal infection particularly arises in immunocompromised patients and those with diabetes mellitus [4,7,10-13]. The main risk factor for this infection is probably the prolonged and profound neutropenia secondary to the myeloablative treatments used for the underlying hematologic malignancy. In fact, mucormycosis occurs more commonly in patients with acute leukemia (78\% according to series of Pagano et al.) than in other types of hematologic malignancies. Further factors, other than neutropenia, associated with increased risk of developing mucormycosis include diabetic ketoacidosis, organ transplantation, HIV disease, drug abuse and haemodialysis. Diabetes mellitus results in impaired chemotaxis and phagocytosis of neutrophils. Treatment with glucocorticoids results in transient T-cell sequestration, diminished synthesis of immunoglobulin, and decreased adherence of neutrophils. These are the major mechanisms responsible for the high incidence of mucormycosis in these patients [1]. In our case although period of neutropenia was short (4 days), she had multiple additional risk factors which were mentioned above. She had a leukemic form of mantle cell lymphoma that caused serious immunosuppression itself, received corticosteroid therapy as a component of chemotherapy regimen. But her blood glucose level was normal during hospitalization. Although she became afebrile and relieved from neutropenia, she deteriorated in the following period rapidly. Similar to our case, a case with high grade stage IV non-Hodgkin lymphoma at remission state was reported previously, who was not neutropenic, did not have fever and expired due to isolated cerebral mucormycosis three weeks after central nervous system prophylaxis [14]. So clinical suspicion is very important.

In 37 cases who developed systemic mucormycosis reported by Italian multicentre study of a large population of patients with hematological malignancy [4], incidence of systemic mucormycosis was about $1 \%$.

Mucorales have high affinity for invading blood vessels (vasculotropism). The invasion of blood vessels leads to thrombosis in the vessels and ischaemia in the involved organ as well as hematogenous dissemination and septic thromboembolism in other organs [1]. In our case, this might explain the lesions which were seen at abdominal CT as multiple small infarctions at spleen, kidneys etc.

Presenting signs and symptoms of mucormycosis are not spesific. In the most frequent pulmonary infection, patient present with fever, cough, thoracic pain and dyspnea [1]. According to involvement sides, symptoms varies like ophthalmoplegia, blindness, proptosis, cornea edema, tissue necrosis in maxillary sinus-retroorbital region involvement or headache, meningism, paresis of cranial nerves in CNS involvement. But it can be completely asymptomatic [1]. In our case, presentation was resembling to acute cholecystitis initially. At follow-up, she was asymptomatic.

The clinical apperance of cutaneous mucormycosis is generally non-spesific. It may arise as a painful erythematous induration of skin resembling common phlebitis. It may progress to a necrotizing lesion with a black eschar [1]. It can be confused with ecthyma gangrenosum which more commonly is caused by Pseudomonas aeruginosa [15]. Our patient also revealed a similar lesion at her malleolus.

The management of this fatal fungal infection begins with establishing diagnosis. The only definite way to diagnose is to visualize the charactheristic hyphae in tissue and materials such as sputum, exudates etc. [1]. In neutropenic patients, antemortem diagnosis is unusual because blood cultures are invaribly negative. Diagnosis is obtained only by invasive methods such as biopsy or surgery but is usually made in less than one-third of patients [2].

Cancer patients, especially patients with leukemia often present pancytopenia including severe thrombocytopenia due to underlying disease or chemotherapy. In these cases resection of the infectious site or biopsy is not feasible [1]. In our patient, biopsy from lesion could not be taken due to this cause.

Therefore, premortal diagnosis of mucormycosis is difficult. Diagnosis has been made only infrequently antemortem [1618]. In a retrospective study on 116 leukemia patients with documented pulmonary filamentous fungal infections, the diagnosis was made ante mortem only in $10 \%$ of patients [4]. In a retrospective study of 37 patients with hematological

www.bjid.com.br 
Figure 1. Ecymotic and bullous lesion on the right malleolus.

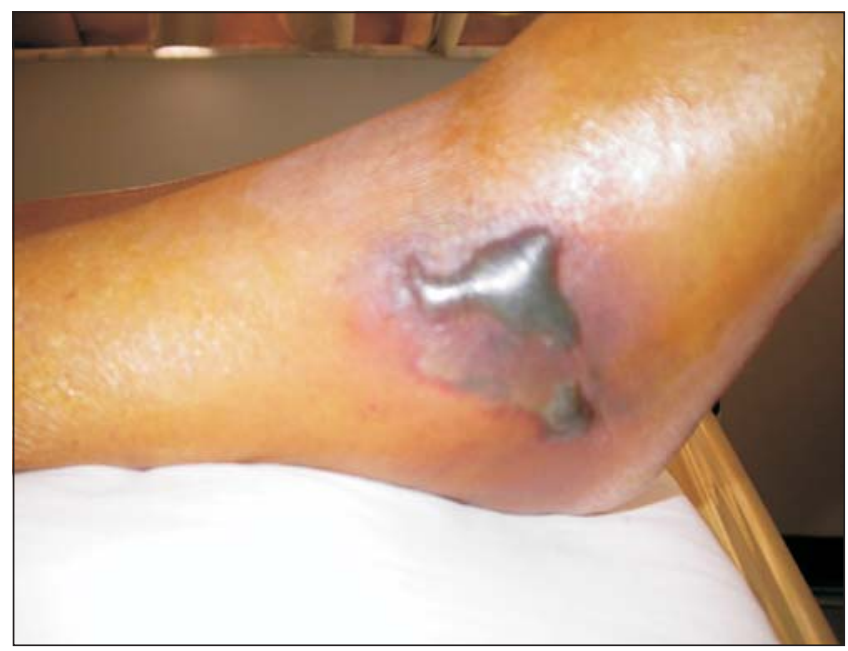

Figure 2. Abdominal CT showing hepatic, splenic and renal infarct.

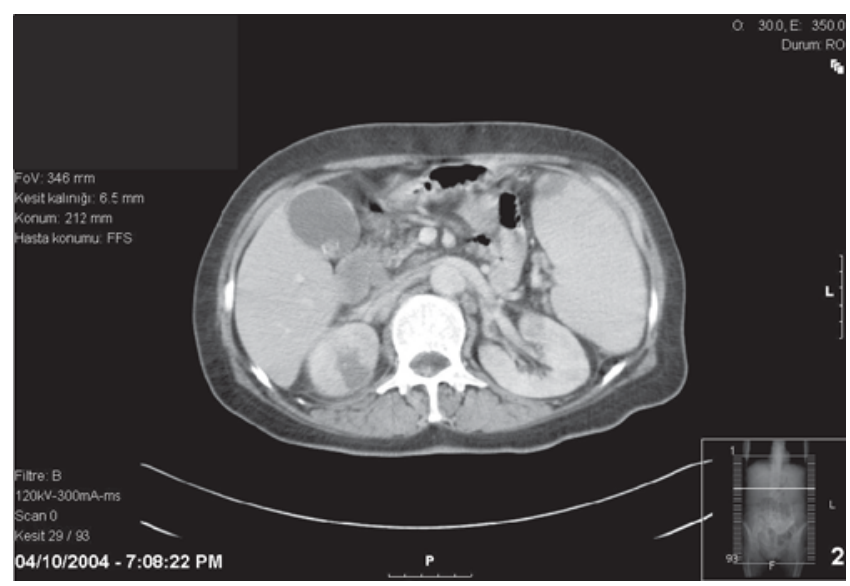

malignancies and histologically documented mucormycosis, the diagnosis was made ante mortem in $35 \%$ of patients [2]. Autopsy case of mucormycosis is seldomly reported. In one large study, disseminated fungal infection was found in $2.55 \%$ (20 cases). Mucormycosis was present in only one case [19]. In another study, systemic fungal infection was found in 27 of 270 autopsies of patients with hematologic malignancies: four of them were mucormycosis infections [20]. We could also diagnosed our patient as having invasive mucormycosis histopathologically by autopsy.

We have encountered only a few reports of cardiac mucor involvement [21-23]. So our case is unusual with the involvement of all vessels of myocardium besides involvement of kidneys, lungs, spleen, diaphragma, submucosa of intestines.

All patients with suspected fungal infection require immediate treatment. Successful treatment of mucormycosis is based on three principles: control of underlying disease, extensive surgical resection of infectious focus or debridement
Figure 3. Necrosis of cardiac muscle and mycotic thrombosis in the lumen of vessel.

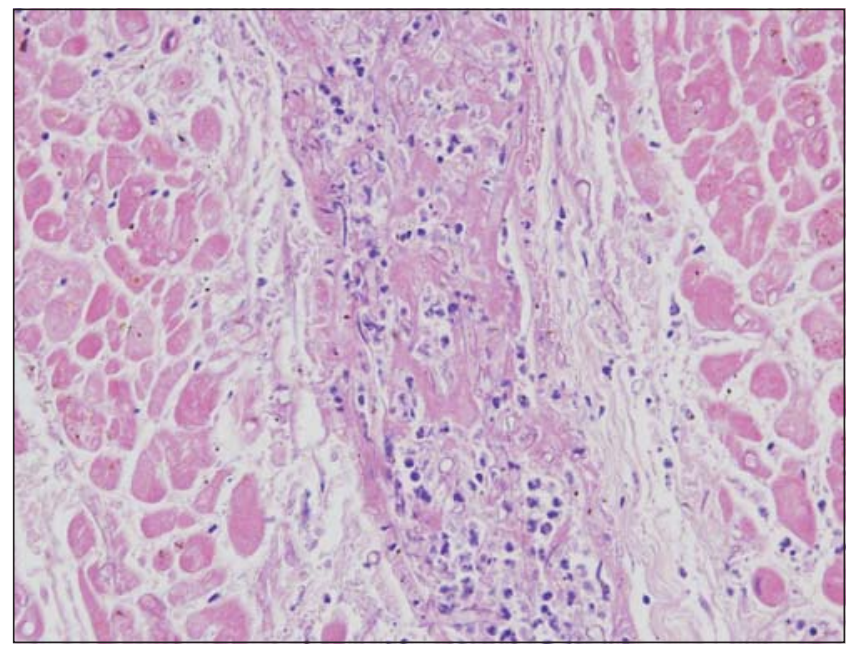

Figure 4. GMS stain showing the fungal structures.

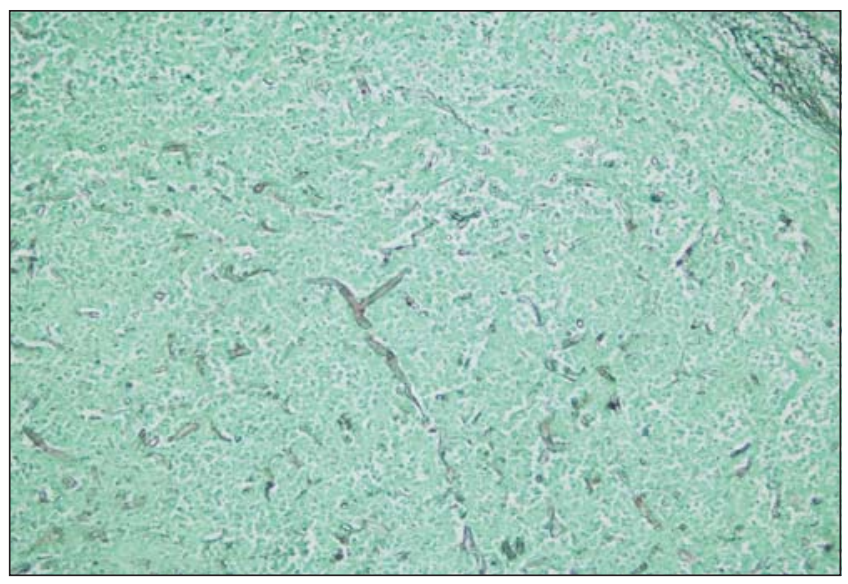

of necrotic tissue and medical treatment with antimycotic agents [1]. But patients with thrombocytopenia are generally not suited for operation if the thrombocyte count cannot be maintained at a high level by peri-operative platelet transfusions. Multiple infectious foci in one organ or disseminated disease are also contraindications and associated with a worse outcome [2]. Intravenous amphotericin B (lipid formulation) is the drug of choice. Recent pharmacokinetic studies demonstrated the safety and tolerance of high doses of L-AmB (15 mg/kg/day). Oral posaconazole is a broad-spectrum oral azole agent that is active in vitro against the zygomycetes and used as step down therapy for patients who have responded to amphotericin B and rarely as salvage therapy for patients who don't respond to or can not tolerate amphotericin B [24]. Therapy should continue until all signs of infection have resolved, and often extends for months. Other antifungal drugs such as caspofungin, variconazole don't seem to add new ammunition against these infections. Since, mucormycosis infection was 
not suspected, we did not begin to antifungal treatment in our patient.

In conclusion, fungal infections should be in mind in case of fever in cases with hematological malignancies whether neutropenia exist or not. Every lesions should be examined very carefully. Biopsy always should be in mind in unexplained lesions. When the fungal infections are thought to exist, antifungal treatment should be begin immediately. So clinical suspicion is very important to treat these severe, fatal fungal infections.

\section{References}

1. Eucker J., Sezer O., Graf B., Possinger K. Mucormycoses. Mycoses 2001;44:253-60.

2. Pagano L., Ricci P., Tonso A. et al. Mucormycosisin patients with haematological malignancies: a retrospective clinical study of 37 cases. Br J Haematol 1997;99:331-6.

3. Pfaffenbach B., Donhuijsen K., Pahnke J. et al. Systemic fungal infections in hematologic neoplasms. An autopsy study of 1,053 patients. Med Klin 1999;89:299-304.

4. Pagano L., Ricci P., Nosari A. et al. Fatal haemoptysis in pulmonary filamentous mycosis: an underevaluated cause of death in patients with acute leukaemia in haematological complete remission. A retrospective study and review of the literature. Gimema Infection Program (Gruppo Italiano Malattie Ematologiche dell’Adulto). Br J Haematol 1995;89:500-5.

5. Sugar A.M. Mucormycosis. Clin Infect Dis 1994;14 Suppl 1:1269.

6. Lehrer R.I., Howard D.H., Sypherd P.S. et al. Mucormycosis. Ann Intern Med 1980;93:93-108.

7. Morrison V.A., McGlave P.B. Mucormycosis in the BMT population. Bone Marrow Transplant 1993;11:383-8.

8. Blin N., Morineau N., Gaillard F. et al. Disseminated mucormycosis associated with invasive pulmonary aspergillosis in a patient treated for post-transplant high-grade non-Hodgkin's lymphoma. Leuk Lymphoma 2004;45(10):2161-3.

9. Pagano L., Offidani M., Fianchi L. et al. Mucormycosis in hematologic patients. Haematologica 2004;89(2):207-14.

10. St-Germain G., Robert A., Ishak M. et al. Infection due to Rhizomucor pusillus: report of four cases in patients with leukemia and review. Clin Infect Dis 1993;16:640-5.
11. Kauffman C.A., Malani A.N. Zygomycosis: an emerging fungal infection with new options for management. Curr Infect Dis Rep 2007;9:435-40.

12. Kontoyiannis D.P., Wessel V.C., Bodey G.P., Rolston K.V. Zygomycosis in the 1990s in a tertiary-care cancer center. Clin Infect Dis 2000;30:851-6.

13. Tonso A., Ricci P., Cenacchi A. et al. Invasive infections by fylamentous mycetes in adult patients with hematological neoplastic diseases (polycentric and retrospective study of 162 cases). Haematologica 1993;78 Suppl 4:24.

14. Zarei M., Morris J., Aachi V. et al. Acute isolated cerebral mucormycosis in a patient with high grade non-Hodgkins lymphoma. Eur J Neurol 2000; 7(4):443-7.

15. Sugar A.M. Agents of mucormycosis and related species. In: Mandell GL, Bennett JE, Dolin R (eds) Principles and practice of infectious diseases. Churchill-Livingstone, Philadelphia, pp 2000:2685-95.

16. Gonzalez C.E., Couriel D.E., Walsh T.J. Disseminated zygomycosis in a neutropenic patient: successful treatment with amphotericin B lipid complex and granulocyte colony-stimulating factor. Clin. Infect. Dis 1997; 24:192-6.

17. St.-Germain G., Robert A., Ishak M. et al. Infection due to Rhizomucor pusillus: report of four cases in patients with leukemia and review. Clin Infect Dis 1993;16:640-5.

18. Rangel-Guerra R.A., Martinez H.R., Saenz C. et al. Rhinocerebral and systemic mucormycosis. Clinical experience with 36 cases. J Neurol Scien 1996;143:19-30.

19. Abe F., Tateyama M., Shibuya H. et al. Disseminated fungal infection. Acta Pathol Jpn 1984;34(6):1201-8.

20. Stahel R.A., Vogt P., Schuler G. et al. Systemic mycoses in hematologic neoplasms. Schweiz Med Wochenschr 1983;113(2):44-6.

21. Tuder R.M. Myocardial infarct in disseminated mucormycosis: case report with special emphasis on the pathogenic mechanisms. Mycopathologia 1985;89(2):81-8.

22. Roy T.M., Anderson K.C., Farrow J.R. Cardiac mucormycosis complicating diabetes mellitus. J Diabet Complications 1990;4(3):132-5.

23. Virmani R., Connor D.H., McAllister H.A. Cardiac mucormycosis. A report of five patients and review of 14 previously reported cases. Am J Clin Pathol 1982;78(1):42-7.

24. van Burik J.A., Hare R.S., Solomon H.F. et al. Posaconazole is effective as salvage therapy in zygomycosis: a retrospective summary of 91 cases. Clin Infect Dis 2006;42:61-5. 